\title{
Evaluación de un programa psico-socio-educativo para hombres que ejercen violencia, en Buenos Aires, Argentina. Marzo de 2021
}

\section{Evaluation of a psycho-socio-educational program for men who exert violence, in Buenos Aires, Argentina. March 2021}

Mario Andrés Payarola ${ }^{a}$

\begin{abstract}
:
A one-year psycho-social-educational program for abusive men developed between the years 2004/2009 in a suburban neighborhood of Buenos Aires, was assessed in order to determine it's efficacy. The assessment was based on individual interviews with the abusive men and separately with their couples (when possible) in the middle (six months) and at the end of the program (twelve months). The features of this intervention model will be explained along with the conclusions of a small sample of such assessment. It will emphasize the cooperative relationship with the clients and also the individual differences in achieving the goals of the program.
\end{abstract}

Keywords:

Gender violence, social problems, PES programs, currency exchange, public policies, efficiency evaluation, group dynamic.

\section{Resumen:}

Se llevó a cabo la evaluación de la eficacia de un programa psico-socio-educativo que funcionó entre los años 2004/2009 en una localidad suburbana cercana a la Ciudad de Buenos Aires, cuya duración era de un año. La misma se realizó a través de entrevistas individuales con los consultantes y en algunos casos separadamente con sus parejas/ex-parejas, a los seis meses y al año de concurrencia de los hombres al programa. Se explicarán las características del modelo de intervención utilizado, como asimismo las conclusiones obtenidas analizando una pequeña muestra de dichas evaluaciones. Se enfatiza en la relación de cooperación establecida con los consultantes y la consideración de sus diferencias individuales para alcanzar los objetivos del programa.

\section{Palabras Clave:}

Violencia de género en la pareja, problema social, Programas P.S.E, cambio, políticas públicas, evaluación de la eficacia, dinámica grupal.

\section{Introducción}

En este artículo disertaremos acerca de cómo hemos evaluado la efectividad de un programa de intervención llevado a cabo entre los años 2005/2009 en un municipio de la zona norte del gran Buenos Aires y expondremos nuestras conclusiones acerca de la teoría del cambio que sustentamos. Proponemos una forma de abordaje (resumida como psico-socio-educativo) basada en un encuadre de grupo pequeño, enfatizando en la dinámica grupal como motor del cambio y poniendo en consideración las características psicológicas de los consultantes. Dicho modelo de intervención con varones que ejercen violencia de género hacia sus parejas mujeres* ${ }^{*}$, surge de la complementación interdisciplinaria de los saberes psicológicos, sociales y educativos, e implicó un intento de superación del modelo sólo "educativo", del que habíamos tomado conocimiento en Canadá, en ocasión de haber obtenido sendas becas de docencia e investigación en dicho país (1996/2000). Asimismo, en cuanto a los contenidos, nuestro modelo se adhiere a las premisas del Modelo de Duluth (Paymar, 2000) y en ese sentido, podría denominarse también como pro-feminista.

\footnotetext{
* De ahora en más se usará esta abreviatura H.V.G para designar: "Hombres que ejercen violencia de género".
}

a Psicólogo, Especialista en Violencia familiar- Fundador de la Red de Equipos de Trabajo y Estudio en Masculinidades, Universidad del Museo Social Argentino, https://orcid.org/0000-0001-7066-6543,Email: mario@ payarola.com.ar 


\section{Método}

Se evaluó la eficacia del modelo psico-socioeducativo, en un programa de asistencia voluntaria para H.V.G. en un Municipio del gran Buenos Aires, entre los años 2004/2009, en donde se llevaron a cabo (sin que se designara ningún colaborador) 240 entrevistas de admisión, lo que derivó en la coordinación de dos grupos abiertos, los que estaban constituidos por diez integrantes cada uno.

\section{Características del programa evaluado}

El formato era de grupo abierto, de manera tal que los consultantes egresaban y se incorporaban nuevos integrantes al producirse nuevas vacantes, luego de una permanencia de aproximadamente un año o más según el caso. Habíamos tenido una experiencia previa en la coordinación de grupos en una ONG, donde no se realizaba evaluación alguna respecto de los progresos de cada integrante, como tampoco se tenía contacto con las parejas o ex parejas, lo que consideramos un errorque no queríamos repetir. Tampoco se había fijado un límite en el tiempo de concurrencia de los participantes lo que resultó inconducente y muchas veces contraproducente.

Determinamos entonces que la duración del programa sería de un año (no rígidamente determinado), es decir, un sujeto podría concurrir algunas veces más antes del egreso, si fuera necesario. La concurrencia a este grupo era voluntaria pues en esos años todavía no se había sancionado la ley de violencia de género (26485) y nunca tuvimos un vínculo formal con el Poder Judicial de la zona, excepto en una ocasión a través del Juzgado de Paz. Asimismo, en esa época aunque existía la ley 24417/1994 (ley de protección contra la violencia familiar) los jueces eran muy reticentes a tomar medidas cautelares (exclusión del hogar) y las víctimas no podían realizar denuncias por su cuenta (salvo en Comisarías), debiendo contar posteriormente con un abogado patrocinante, lo que dificultaba su acceso a la justicia. El programa dependía de un Centro de atención a mujeres víctimas de violencia, cuya directora aprobó la iniciativa de crearlo. Se estableció un lazo de interconsulta permanente entre el programa de atención a mujeres y la institución oficial de defensa de los derechos de niñas/niños y adolescentes. Ambas instituciones estaban muy cerca geográficamente y ellas mismas realizaban derivaciones al grupo P.S.E, como también consultas sobre determinados casos en común (la mujer era atendida en el centro de la mujer y el varón en nuestro programa).

No siendo de asistencia obligatoria, los concurrentes realizaban una consulta de manera voluntaria al momento de estar en crisis con su pareja, generalmente cuando éstas le decían: "O te tratas o te vas", o ellos expresaban en la entrevista de admisión "vengo para unir a la pareja" o "vengo para unir a la familia". Se les aclaraba que ese no era el propósito del programa y que el objetivo del mismo era ayudarlos a dejar de ejercer violencia. En general, presentaban las características del hombre violento "cíclico" (Walker, 1979).

Antes de ser derivados al grupo, se tomaba una entrevista de admisión, siendo rechazadas aquellas personalidades con características "psicopáticas", quienes presentaban problemas psiquiátricos severos y aquellos que presentaban consumo problemático de sustancias y no estaban en tratamiento específico para superarlo. En todos los casos se procedía a la derivación correspondiente en las instituciones de la zona (Hospital General, Alcohólicos Anónimos, etc.).

Al ser incorporados al grupo se les hacía firmar un consentimiento en el que se les informaba que el coordinador podría comunicarse con sus parejas, ex parejas en caso de que evaluara que ella o sus hijos pudieran estar en riesgo. En caso que no lo fuera, ellas serían citadas a los seis meses y al año a los efectos de una evaluación, siempre y cuando ellas quisieran colaborar. Ninguno se negó a firmar dicho consentimiento.

Al momento de ingresar al grupo se los invitaba a comprar un ejemplar del libro "Aprender a vivir sin violencia", Sonkin y Durphy (1997) y empezaran a practicar el "tiempo afuera" y el diario del enojo, siendo el primero más utilizado que el segundo.

\section{La dinámica grupal planteada por los grupos P.S.E.}

En todos los programas conocidos, incluso en el nuestro P.S.E. enfatizamos en la necesidad de brindar seguridad a las víctimas (mujer e hijos) del consultante, por lo cual los coordinadores deben evitar aquellas intervenciones que podrían incrementar el riesgo para los mencionados. Por tal motivo se debe partir de la consigna de "nuncajustificar la violencia", y no aceptar ninguna de las excusas que normalmente esgrimen los varones al momento de iniciar el grupo (Paymar, 2000). Seguidamente, desarrollamos una secuencia de discusión/confrontación grupal, con reuniones semanales, durante una hora y media. Generalmente en las primeras reuniones, se trata de que se responsabilicen por la violencia ejercida, aprendan a detener la violencia física, y revisen los aspectos que constituyen el perfil psicológico del H.V.G. (Dohmen, M., 1995), para posteriormente finalizar con el cuestionamiento de los estereotipos de género (Paymar, 2000) y de esta manera consolidar el cambio. 


\section{Resultados}

Las respuestas a las preguntas realizadas a 15 varones luego de pueden resumirse así.

1. Aprendieron a responsabilizarse por sus conductas violentas.

2. Aprendieron que la violencia no es sólo física.

3. Aprendieron lasseñales de pre-violencia, y adoptaron el "tiempo afuera".

4. Aprendieron a discutir abiertamente en el grupo los problemas de relación que tienen con sus parejas.

5. Mencionan que cuando faltan al grupo por algún motivo, lo extrañan.

6. Reconocen que en el grupo pueden hablar temas que no podrían en otro lado.

7. Reconocen que sus compañeros no son más violentos que él (aprendieron a no externalizar ni minimizar la gravedad de sus propias conductas violentas).

\section{Segunda parte de la evaluación semestral}

Finalmente, una pregunta abierta inquiere acerca de qué es lo que les falta cambiar y cuyas respuestas tienen los siguientes factores en común:

1. Comunicación: modificar la forma de comunicarse con su pareja, admitiendo que lo hacen de mala manera (levantando el tono de voz), en forma de reproches, poder decir las cosas que le molestan sin agredir/ofender (Comunicación asertiva).

2. Parentalidad: problemas de relación con sus hijos, a los cuales no le tienen paciencia, y desean aprender a poner límites a los niños sin malos tratos.

3. Ejercitar la tolerancia y seguir trabajando para no escalar en las discusiones con sus parejas.

4. Actitudes posesivas/celos: trabajar sus miedos e inseguridad respecto a su pareja.

5. Aceptar los errores cometidos en la relación y tratar de repararlos.

6. Ser más compañero.

7. Respetar las decisiones tomadas por su pareja.

8. Expresar sus sentimientos y ser más afectivo.

9. Modificar la rigidez de conducta.

10. Poder hablar de su propia infancia y de la relación con sus padres.

Respuestas de las mujeres a los seis meses de concurrencia de sus parejas al grupo (resumen):
1. Su pareja se toma el tiempo afuera, no tiene más explosiones de violencia, no rompe cosas y no ha habido violencia física desde que concure al grupo.

2. Está poniendo compromiso en cambiar.

3. Llega mejor después del grupo, cuenta lo que trabaja en el grupo, está contento.

4. Escucha más, está más comprensivo.

5. Por momentos profiere insultos, pero no con la misma frecuencia y no dirigidos hacia ella.

6. La sigue controlando le revisa todo, manipulador, mentiroso, no obstante, lo quiere mucho y no quiere separarse (Respuesta preocupante).

7. Aprendió a ser violento de manera más sutil (Respuesta preocupante).

8. Pone esfuerzo en relacionarse mejor con su hijo, pero no con ella. Esta se quiere separar y él no. (El coordinador le solicitó al consultante que dejara de concurrir al grupo y que se siga atendiendo con una psiquiatra quien lo medicaría, al no poder adaptarse a la dinámica grupal).

9. Empezó a preocuparse por ellay a dialogar más, se toma tiempo afuera, se generan discusiones por dinero, ella también implementa el tiempo afuera.

10. No tiene violencia verbal, reconoce que se puso celoso en relación a los horarios de trabajo de ella. Lo nota más sincero.

\section{Discusión}

Lamento defraudar al lector que haya esperado que este artículo arrojara algún porcentaje de recuperación como para luego concluir las bondades del modelo presentado en comparación con otros. Por el contrario, nuestra intención es demostrar que pueden llevarse a cabo evaluaciones con los mismos consultantes y darles lugar a opinar acerca del proceso que llevan a cabo y explicarles lo que pueden esperar del mismo. También aclaramos que no estamos de acuerdo con la tan reiterada palabra utilizada para estos hombres "recuperación", pues esto indicaría que el consultante recuperaría una capacidad que había perdido. Por otro lado, se utiliza frecuentemente las estadísticas de reincidencia para medir la eficacia o no de un programa. Esto sucede cuando los programas ya están instalados en un determinado contexto y funcionan de manera masiva. Generalmente, ya están regulados rígidamente en tamaño de duración y contenidos a impartir y donde es posible hacer un seguimiento longitudinal de los casos.

Desde nuestro punto de vista, (que creemos compartido con muchos colegas), en realidad en nuestros programas, el sujeto adquiere/aprende, habilidades en la 
dinámica grupal que antes no poseía, las que le permitirán relacionarse sin violencia hacia su pareja e hijos. Aunque nuestro programa P.S.E. establece objetivos, el aprendizaje no se realiza de manera lineal (como lo proponen los modelos sólo educativos y generalmente en un tiempo muy acotado) sino por el contrario, en un proceso dialéctico con avances y retrocesos hasta su definitiva consolidación (Stefanakis, 1996).

En nuestro ejemplo, resaltamos la posibilidad de realizar evaluaciones a mitad del proceso y al final del mismo. En relación a la primera, esto permite realizar las correcciones necesarias en aquellos casos en que no hayan podido alcanzar los objetivos propuestos y no teniendo que esperar el final de proceso para tomar una acción diferente con esos consultantes.

La designación de grupo P.S.E. responde a la hipótesis que nuestros consultantes no son considerados enfermos y no poseen una problemática psicológica/psiquiátrica, por lo tanto, no se designará con el nombre de grupo psicoterapéutico. Aunque es necesario aclarar que se utilizan herramientas de distintos enfoques (eclecticismo) que originalmente fueron diseñados para ámbitos terapéuticos. Tampoco es un "grupo reflexivo", pues su concurrencia no pretende sólo reflexionar y no son "talleres", porque no pretenden solamente sensibilizar a los concurrentes con la problemática de violencia. Sin embargo ambos tipos de intervenciones son utilizadas frecuentemente durante el desarrollo de los grupos P.S.E (Teubal, 2005), conjuntamente con otras (P.ej. psicodrama).

\section{Limitaciones}

Como se ha dicho, el programa estaba coordinado por una sola persona, al no haberse designado colaboradores a medida que fue creciendo la cantidad de consultas. Asimismo, el hecho de que no hubiera leyes que determinaran con claridad las situaciones de violencia de género (como la que fue sancionada el 11 de marzo de 2009 , ley 26.485), la violencia era considerada en ese entonces como algo perteneciente al ámbito privado.

Como pionero en el tema que nos ocupa, la creación de la Red de Equipos de Trabajo y Estudio en Masculinidades (RETEM) en 2011 fue el paso inicial para romper con el aislamiento de quienes trabajaban en otros programas de intervención, propiciando un ámbito de discusión teórica respecto de la especialidad y expandiendo el surgimiento de nuevos programas en diferentes localidades.

Es necesario aclarar, que contrariamente al programa explicado, en la actualidad a partir de la sanción de esas nuevas leyes tanto a nivel provincial como Nacional, la mayoría de los consultantes son derivados por la ju sticia (civil/penal), lo que en la práctica redunda en una falta de motivación inicial para el ingreso del consultante al programa. Asimismo, los jueces tienen la potestad de imponer ciertas pautas de conducta además de la concurrencia obligatoria al programa.

\section{Conclusiones}

Hemos visto en este artículo la complejidad de fuerzas que intervienen en las manifestaciones de la violencia de género en la pareja, desde lo macro, exo y micro-sistémico y las intervenciones posibles para prevenirla. El desafío es desandar dicha complejidad, aportando claridad desde las intervenciones con varones H.V.G. de manera grupal. La posibilidad de desarrollar programas P.S.E. es una alternativa que en general resulta eficaz en la obtención de los objetivos trazados, pues la dinámica grupal se transforma en herramienta de cambio, al generarse una cohesión que permite la contención y confrontación de ideas de manera respetuosa, para producirlo. La evaluación de la eficacia aportada como ejemplo, en un servicio llevado a cabo durante cinco años, con el encuadre de grupo abierto, demuestra que es posible hacer una evaluación a los seis meses y al año de concurrencia, en entrevistas individuales con cada consultante. Esto permite observar de manera personalizada el grado de compromiso y aprendizaje de conductas alternativas al uso de la violencia. Asimismo se le brinda un espacio para que plantee sus propias necesidades de atención.

Todo ello, con tribuiría a generar un compromiso para el cambio, reduciendo el riesgo de violencia evaluada en la entrevista inicial y evitando la reincidencia en el futuro. Propongo que el sostenimiento en dichas nuevas conductas aprendidas podría ser estable en el tiempo si se crea una instancia de monitoreo o seguimiento a lo largo de un cierto período de tiempo, como también invitar a los consultantes a comunicarse con el servicio si tienen preocupación por alguna situación en la que vislumbren la posibilidad de reincidir en una conducta violenta.

En relación al tiempo de concurrencia, se ha demostrado con la evaluación llevada a cabo, que un año (52 semanas), concurriendo una vez por semana durante una hora y media, es un tiempo razonable para lograr los cambios propuestos con este tipo de población y con este tipo de intervención. No obstante, el mensaje a trasmitir hacia las víctimas, en cuanto a las posibilidades de cambio de los varones debiera ser prudente, instando a las mujeres a que no dejen de recibir apoyo/contención/protección mientras que el varón está concurriendo al grupo.

Como lo señalé anteriormente, la evaluación de la efectividad de los programas debe tener en cuenta la población a atender y el contexto social particular en que se desenvuelve, la comu nidad, las leyes que rigen en ese país/provincia, etc. (Modelo ecológico), como también las características individuales de cada persona y su historia 
personal. Asimismo, no ignoramos que en nuestro país la violencia de género coexiste con otras vulnerabilidades sociales (falta de empleo, vivienda digna, recursos económicos, etc.) lo que merece una adecuación de los encuadres a dicha realidad, según la comunidad que se trate. La falta de una política pública en la Argentina respecto de las intervenciones con mujeres y los H.V.G. agrega un elemento de inestabilidad laboral a los que tienen a cargo estos dispositivos y dificulta la permanencia de los mismos en el tiempo. A su vez, esto último impide también la posibilidad de llevar a cabo investigaciones longitudinales con quienes concurren al programa durante un cierto período de tiempo.

Finalmente, creemos que los modelos sólo educativos al ser tan rígidos, no permiten una interacción entre coordinadores y grupo, y posteriormente, se dificulta evaluar el grado de aprovechamiento individual de los contenidos impartidos. Por el contrario, en los grupos P.S.E. las características de la dinámica grupal generada entre los coordinadores/grupo e integrantes entre sí es favorecedora del cambio, como lo demuestra el ejemplo aquí aportado, como también la bibliografía especializada en las problemáticas sociales (Hivos, 2014; Geldschläger, Gines y Ponce, 2011; Teubal, 2005). En nuestro medio, contamos con el antecedente y la producción bibliográfica del Dr. E. Pichón Riviere, $(1980,1985)$ cuya herramienta de cambio es el grupo (dinámica grupal y vínculo) el que es aplicado para un a diversidad de problemáticas sociales (ver bibliografía), entre ellas la violencia de género en la pareja.

\section{Referencias}

Dohmen, M. (1995). Perfil del hombre golpeador, monografía sin publicar, Buenos Aires, Argentina.

Geldschäger, H., Ginés, O. y Ponce, A. (2011). Grupo psicoterapéutico con hombres que ejercen violencia de género. En: Neus, R., Júlia, M (Ed.). Intervención grupal en violencia sexista experiencia, investigación y evaluación ( $\mathrm{pp}$ 344-368). Herder.

Hivos (2014).Gender and Theories of Change, 4th Edition, Ediscussigon. People unlimited.

Ley 26485/2009 - Ley de Protección Integral para prevenir sancionar y erradicar la violencia contra las mujeres en los ámbitos en que desarrollen sus relaciones interpersonales. Sancionada el 11 de marzo de 2009, promulgada de hecho el 1 de abril de 2009.

Paymar, M. (2000). Violence no more, Hunter Publishers, Alameda, CA, U.S.A

Pichon Rivière, E. (1980). Teoría del vínculo. Buenos Aires, Argentina: Nueva Visión.

Pichón Riviére, E. (1985). El proceso grupal: Del psicoanálisis a la Psicología Social, Buenos Aires, Argentina. Nueva Visión.

Sonkin y Durphy (1997). Aprender a vivirsin violencia, USA. Vulcano Press.

Stefanakis, H. (1996). Modelo trans-teórico de cambio: Un modelo heurístico para la exploración acerca de cómo terminar con la violencia de hombre hacia la mujer: 1era parte. En: Mario, A, P. (Trad.), Trabajando con hombres. New Haven
Correctional Centre, USA (Obra original publicada en 1996)

Teubal, R. (2005). Factores de cambio en los grupos y aportes para la intervención, Revista Topia, Abril de 2005. https://www.topia.com.ar/articulos/factores-de-cambioen-los-grupos-y-aportes-para-la-intervenci\%C3\%B3n.

Walker, L. (1979). The Battered woman, Harper \& Row, New York. U.S.A. 